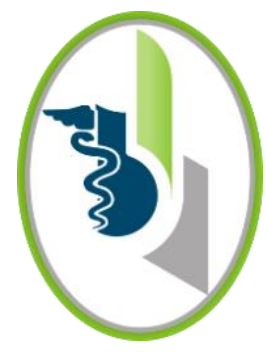

ACCESO abierto

Para citaciones: Chávez, M., García, L., Chaves, J., Duran, K., Ramírez, J. (2020). Prevalencia de infecciones vaginales en mujeres embarazadas y no embarazadas en un hospital de Cali, Colombia. Revista Ciencias Biomédicas, 9(2), 92-102.

Recibido: 13 de marzo de 2019 Aprobado: 28 de enero de 2020

Autor de correspondencia:

Mónica Chávez Vivas

monikchavez@gmail.com

Editor: Inés Benedetti. Universidad de Cartagena-Colombia.

Copyright: (C) 2020. Chávez, M., García, L., Chave, J., Duran, K., Ramírez, J. Este es un artículo de acceso abierto, distribuido bajo los términos de la licencia https://creativecommons.org/licenses/bync-sa/4.0/ la cual permite el uso sin restricciones, distribución y reproducción en cualquier medio, siempre y cuando el original, el autor y la fuente sean acreditados.

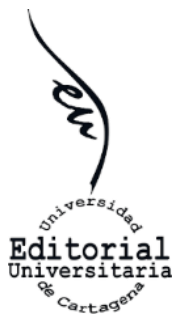

\section{Prevalencia de infecciones vaginales en mujeres embarazadas y no embarazadas en un hospital de Cali, Colombia}

\author{
Prevalence of vaginal infections in pregnant and non-pregnant \\ women in a hospital of Cali, Colombia.
}
Mónica Chávez Vivas ${ }^{1}$, Laura García Blanco², Jenny Chaves Sotelo ${ }^{3}$, Katherine Duran ${ }^{3}$, Jeniffer Ramírez ${ }^{4}$

\author{
1 Departamento de Ciencias Biomédicas. Facultad de Salud. Universidad Santiago de Cali. Centro de \\ Investigación y Estudios CEIS. Grupo de Investigación GEFIME, Cali, Colombia. \\ ${ }^{2}$ Hospital Primitivo Iglesias, Cali, Colombia. \\ ${ }^{3}$ Hospital San Juan de Dios, Cali, Colombia. \\ ${ }^{4}$ Hospital Local de Vijes, Vijes, Colombia.
}

\section{RESUMEN}

Introducción: las infecciones vaginales son frecuentes en todos los grupos de edad, pueden presentar una manifestación asintomática, compromiso local o sistémico.

Objetivo: establecer la prevalencia de infecciones vaginales en mujeres embarazadas y no embarazadas que consultaron a un hospital de mediana complejidad de la ciudad de Cali.

Métodos: se realizó un estudio descriptivo y retrospectivo basado en los reportes de las historias clínicas de pacientes con diagnóstico de vulvovaginitis infecciosa durante un período de un año. Las variables fueron evaluadas estadísticamente para determinar su grado de significancia en la patología..

Resultados: se encontraron 534 registros con una edad promedio de 24 años ( $\mathrm{DS}=10,553$; edad min=14 años, máx.= 80 años) entre las mujeres que consultaron por infección vaginal. La vaginosis bacteriana fue más prevalente con un $18.5 \%$, seguida de la vaginitis candidiásica (15.7\%). Las mujeres diagnosticadas con vaginitis candidiásica presentaron un mayor riesgo de desarrollar hemorragias (1.1 $\%$; OR: $3.436 ; p=0.016)$ y neoplasias $(2.4 \%$; OR $2.278 ; p=0.014)$. Las mujeres diagnosticadas con vaginosis bacteriana se relacionaron con un mayor riesgo de desarrollar enfermedad inflamatoria pélvica (2.2\%; OR: 28.286). La prevalencia de mujeres embarazadas con infecciones vaginales fue del $20.8 \%$, siendo la vaginitis candidiásica el motivo por el cual consultaron con mayor frecuencia.

Conclusiones: en este estudio se demostró que las infecciones vaginales afectan a las mujeres en edades reproductivas y sus principales complicaciones derivan en la enfermedad inflamatoria pélvica, hemorragias y neoplasias; con predominio de la vaginosis bacteriana, especialmente, en mujeres de estratos socio-económicamente bajos como es nuestro grupo de estudio.

Palabras Clave: vulvovaginitis; vaginitis; vaginosis bacteriana; Trichomona vaginalis; Candida albicans; Gardnerella vaginalis. 


\begin{abstract}
Introduction: vaginal infections are common in all age groups may present an asymptomatic manifestation or local and systemic involvement.
\end{abstract}

Objective: establish the prevalence of vaginal infections in pregnant women and nonpregnant who consulted a medium complexity hospital in the city of Cali.

Methods: a descriptive and retrospective study was conducted from clinical records of patients diagnosed with infectious vulvovaginitis in 2014. Variables were statistically evaluated to determine the degree of significance in pathology.

Results: a total of 534 records were analyzed with an average age of 24 years (SD = 10.553; age $=14$ years $\min , \max =80$ years) among women who consulted for vaginal infection. Bacterial vaginosis was more prevalent with un $18.5 \%$, followed by Candida vaginitis (15.7\%). Women diagnosed with candidal vaginitis had a higher risk of bleeding (1.8\%; OR: 3.436; $\mathrm{p}=0.016)$ and malignancies $(3.9 \%$; OR $2.278 ; \mathrm{p}=0.014)$. Women diagnosed with candida vaginitis had a higher risk of developing bleeding (1.8\%; OR: 3.436; $\mathrm{p}=0.016)$ and malignancies $(3.9 \%$; OR $2.278 ; \mathrm{p}=0.014)$. Women diagnosed with bacterial vaginosis were associated with an increased risk of developing pelvic inflammatory disease (3.6\%; OR: 28.286). The prevalence of pregnant women with vaginal infections was $20.8 \%$, the Candida vaginitis the why most frequently consulted.

Conclusion: this study showed that vaginal infections affect women of reproductive age and their main complications were pelvic inflammatory disease, hemorrhage and cancers, especially in women with bacterial vaginosis and lower socio-economically strata.

Keywords: vulvovaginitis; vaginitis; bacterial vaginosis; Trichomonas vaginalis; Candida albicans; Gardnerella vaginalis.

\section{INTRODUCCIÓN}

Las infecciones vaginales son un conjunto de entidades ginecológicas relativamente frecuentes, y caracterizadas por la alteración de la flora vaginal normal con disminución de lactobacilos y aumento de agentes infecciosos que abarcan virus, bacterias, protozoarios y hongos (1). Aunque las infecciones pueden presentarse asociadas con flujo vaginal, mal olor, dolor, ardor y prurito, no todas son sintomáticas y su prevalencia real es difícil de establecer en la comunidad $(1,2)$. Sin embargo, algunos estudios estiman que la prevalencia de vulvovaginitis puede variar entre 5 y $26 \%$ en embarazadas $(3,4)$, y entre 24 y $37 \%$ en mujeres atendidas en clínicas con servicios de atención de infecciones de transmisión sexual en diferentes lugares del mundo (ITS) $(5,6)$.
Entre las infecciones vaginales, la vaginosis bacteriana es la más común en mujeres en edad reproductiva $(7,8)$. Hasta un $50 \%$ de las pacientes pueden cursar asintomáticas; sin embargo, es la patología que representa aproximadamente la tercera parte de todos los casos de vulvovaginitis (7). Las prevalencias reportadas de este tipo de infección son muy variables, es así como se reportan desde un 5\% en adolescentes hasta llegar a representar un $50 \%$ en mujeres en edad reproductiva de zonas rurales de India (6). Otra de las infecciones vaginales reportadas frecuentemente es la debida a levaduras del género Candida, principalmente Candida albicans $(9,10)$. Sin embargo, otras especies de Candida han emergido como $C$. glabrata, $C$. parapsilosis, y $C$. tropicalis (11). Se estima que hasta un $75 \%$ de las mujeres sexualmente activas sufren candidiasis vaginal al menos una vez en la vida y entre el 5 a 
$10 \%$ de ellas la padecen en forma recurrente (tres o más episodios en un año) $(9,12)$. Por otra parte, se estima que la tricomoniasis afecta a 170 millones de mujeres anualmente en todo el mundo (13). Esta infección, causada por Trichomona vaginalis, un protozoo flagelado amitocondrial, produce sintomatología clínica similar a la de otros microorganismos como Candida spp y Gardnerella vaginalis $(14,15)$.

Algunas mujeres conviven con estas infecciones y en ocasiones pasan inadvertidas, pero durante el embarazo esto constituye un grave problema, debido a que representan un factor de riesgo para sufrir complicaciones obstétricas $(3,4)$. El diagnóstico certero es la base para evitar posibles complicaciones como la enfermedad inflamatoria pelviana y las complicaciones del embarazo $(16,17)$.

En la práctica clínica, las infecciones vaginales se diagnostican de acuerdo a la sintomatología y características del flujo vaginal y, la mayoría de veces, se inicia un tratamiento empírico, lo que lleva a que las pacientes no consulten al especialista y se automediquen causando así mayor incidencia de otras enfermedades de transmisión sexual (ETS) (17). Una mayor comprensión de la epidemiología de la infección posibilita el establecimiento de medidas eficientes en el manejo racional de las infecciones que produce, lo cual redunda en el mejoramiento de las condiciones de salud de la población. El objetivo de este estudio fue establecer la prevalencia de infecciones vaginales en mujeres embarazadas y no embarazadas que consultaron a un hospital de mediana complejidad de la ciudad de Cali, Colombia.

\section{MÉTODOS}

Se realizó un estudio descriptivo y retrospectivo de prevalencia, donde se incluyeron las pacientes mujeres embarazadas y no embarazadas que consultaron al hospital San Juan de Dios de la ciudad de Cali por presentar flujo vaginal acompañado de síntomas a nivel vaginal (prurito, hinchazón, dificultad para realizar actividad sexual, enrojecimiento vaginal o placas) durante un período de dos años. El hospital San Juan de Dios de la ciudad de Cali sirve a la población de bajos recursos económicos y realiza consulta ginecológica $\mathrm{y}$ obstétrica de primer y segundo nivel. Los datos se obtuvieron a partir de historias clínicas y se seleccionaron las mujeres que no habían recibido tratamiento antimicrobiano en los últimos tres meses previo al estudio.

Se revisaron los reportes con resultados completos del diagnóstico clínico y el análisis microbiológico que se realizó en el laboratorio clínico del hospital. Para este fin, la observación microscópica y la medida del $\mathrm{pH}$ del exudado vaginal fueron los criterios diagnósticos para establecer la vulvovaginitis $(5,13)$. El diagnóstico de vaginosis bacteriana se basó en la presencia de al menos tres de los cuatro criterios clínicos propuestos por Amsel y col (18), los cuales han sido aceptadas como parámetro para indicar la presencia de la enfermedad. El criterio diagnóstico para la identificación de vulvovaginitis causada por Candida $s p$, se basó en la presencia de leucocitos, células epiteliales, levaduras y pseudomicelios en el examen microscópico y un del exudado vaginal $\mathrm{pH}$ $<4.5(5,17)$. En el caso del diagnóstico de la tricomoniasis, la evaluación se basó en la observación de leucocitos y Trichomonas $s p$. con un $\mathrm{pH}>$ de $4.5(5,13)$.

En esta investigación se tomaron los datos a partir de las historias clínicas con previo aval del comité científico y de ética del hospital. Los datos se manejaron por códigos para guardar la confidencialidad de las pacientes y preservar siempre la veracidad de la información.

\section{Análisis estadístico}

La unidad de análisis fue número de mujeres diagnosticadas con infecciones vaginales, la edad de la paciente, resultado de los estudios microbiológicos, hallazgos clínicos, comorbilidades y paciente en estado de embarazo o no. Se construyó una base de datos con las variables de interés, empleando el programa Excel ${ }^{\circledR}$. 
Los valores de las variables cuantitativas fueron expresados como porcentaje relativo de la población. La población de estudio se separó en grupos de mujeres embarazadas y no embarazadas. La presencia de los patógenos fue determinada como porcentaje teniendo en cuenta la edad de la paciente, antecedentes médicos como diabetes $\mathrm{u}$ otras entidades, uso de anovulatorios orales, características paraclínicas y comorbilidad presentada, en el caso de las mujeres embarazadas se reportó el trimestre del embarazo relacionado con la infección. Se realizó un análisis de asociación entre las distintas variables y la significancia estadística en las diferencias en la frecuencia de las variables entre los grupos establecidos fue determinada por análisis estadístico, empleando la prueba de chi-cuadrado de Pearson con valores de $\mathrm{p}<0,05$, considerando un nivel de confianza del $95 \%$ (alfa) y un error (beta) del 5\%. El paquete estadístico que se empleó para este fin fue el SPSS vs 22.0 (Inc, Chicago) siguiendo los estándares de la guía SAMPLE (19).

\section{RESULTADOS}

Las mujeres que consultaron al hospital por infección vaginal correspondieron a 534 pacientes con un promedio de edad de 24 años $( \pm \mathrm{DS}=10,55$; edad min=14años, máx. $=80$ años). El rango de edad entre 20 y 40 años fue el más frecuente en los registros de las consultas, con un $81,8 \%$ de prevalencia y en este grupo la vaginosis bacteriana fue la más prevalente (18.2\%). Las mujeres mayores de 41 años presentaron el menor número de reportes de infecciones vaginales. Sin embargo, las mujeres con edades entre 41 y 64 años presentaron un mayor riesgo de contraer vaginosis bacteriana de una forma significativa $(\mathrm{OR}=2.575 ; \mathrm{p}=0.010),($ tabla 1$)$.

La vaginosis de tipo bacteriano fue la más prevalente con 99 casos (18.5\%), seguida por la vaginitis candidiásica con 84 casos $(15.7 \%)$, la vaginitis causada por Trichomona vaginalis representó el $2.7 \%$ de los casos. Otros motivos de consulta estuvieron representados por, cervicitis $(4.3 \%)$ e infecciones por otras bacterias como: Corynecbacterium spp., cocobacilos variables, bacilos Gram positivos y Lactobacillus sp. Se encontraron $95(17.8 \%)$ reportes de infecciones vaginales en adolescentes, siendo la vaginitis candidiásica la más prevalente $(4.1 \%)$.

Tabla 1. Distribución de vaginitis candidiásica y vaginosis bacteriana entre los grupos etarios de las mujeres evaluadas

\begin{tabular}{ccccccccc}
\hline \multicolumn{7}{c}{ Vaginitis candidiásica } & \multicolumn{5}{c}{ Vaginosis bacteriana } \\
\hline $\begin{array}{l}\text { Grupo } \\
\text { etario } \\
(\text { años })\end{array}$ & $\mathrm{n}(\%)$ & OR & $\begin{array}{c}\text { IC } \\
\text { Min- Max }\end{array}$ & $\mathrm{p}$ & $\mathrm{n}(\%)$ & $\mathrm{OR}^{*}$ & $\begin{array}{c}\text { IC } \\
\text { Min- Max }\end{array}$ & $p^{\dagger}$ \\
\hline $14-19$ & $19(5.7)$ & 1.465 & $0.830-2.586$ & 0.186 & $19(5.7)$ & 1.057 & $0.606-18843$ & 0.846 \\
\hline $20-40$ & $53(16.1)$ & 1.433 & $0.883-2.327$ & 0.144 & $60(18.2)$ & 1.090 & $0.705-1.684$ & 0.699 \\
\hline $41-64$ & $4(1.2)$ & 0.737 & $0.252-2.154$ & 0.575 & $12(3.6)$ & 2.575 & $1.223-5.422$ & 0.010 \\
\hline$>65$ & 0 & 0 & 0 & - & $2(0.6)$ & 0.412 & $0.083-2.041$ & 0.370 \\
\hline$*$ OR=Odds Ratio & & & & & &
\end{tabular}


Otro hallazgo importante se relaciona con las mujeres diagnosticadas con vaginitis candidiásica, las cuales se asociaron con un mayor riesgo de desarrollar hemorragias (1.8 \%; OR: 3.436; $\mathrm{p}=0$.
016) y neoplasias (3.9\%; OR 2.278; $\mathrm{P}=0.014)$ en forma significativa (tabla 2).

Tabla 2. Relación entre vaginitis candidiásica, vaginosis bacteriana y presencia de otras comorbilidades, $\mathbf{n}=\mathbf{5 3 4}$

\begin{tabular}{lcccccccc}
\hline & \multicolumn{7}{c}{ Diagnóstico } \\
\cline { 2 - 9 } Comorbilidades & \multicolumn{7}{c}{ Vaginitis Candidiásica } & \multicolumn{4}{c}{ Vaginosis bacteriana } \\
\cline { 2 - 9 } & $\mathrm{n}$ & OR & IC & $\mathrm{p}$ & $\mathrm{n}$ & OR & IC & $p$ \\
& $(\%)$ & & Min-Max & & $(\%)$ & & Min-Max & \\
\hline Neoplasia & 13 & 2.278 & $1.145-4.530$ & 0.016 & 29 & 8.992 & $4.751-17.019$ & 0.000 \\
& $(2.4)$ & & & & $(5.4)$ & & & \\
\hline EPI & 2 & 0.903 & $0.198-4.112$ & 0.895 & 12 & 28.286 & $6.224-128.548$ & 0.000 \\
& $(0.37)$ & & & & $(2.2)$ & & & \\
\hline Hemorragia & 6 & 3.436 & $1.214-9.729$ & 0.014 & 7 & 3.419 & $1.242-9.409$ & 0.012 \\
& $(1,1)$ & & & & $(1.3)$ & & & \\
\hline ITU & 4 & 1.852 & $0.583-5.889$ & 0.289 & 3 & 0.965 & $0.270-3.449$ & 0.956 \\
& $(0,74)$ & & & & $(0.6)$ & & & \\
\hline
\end{tabular}

$\mathrm{EPI}=$ Enfermedad pélvica inflamatoria; ITU= Infección del tracto urinario. $P=$ error con índice de confianza del $95 \%$

En el caso de las mujeres diagnosticadas con vaginosis bacteriana los resultados indican que el $2.2 \%$ presentan alto riesgo de desarrollar enfermedad pélvica inflamatoria (EPI) (OR: 28.286; $\mathrm{P}<0.05)$ ), el $5.4 \%$ tenían ocho veces más riesgo de desarrollar neoplasias $(\mathrm{OR}=8.992 ; \mathrm{P}<0.05)$ y el $1.3 \%$ de los casos tenían un riesgo mayor a tres de presentar hemorragias $(\mathrm{OR}=3.419 ; \mathrm{P}=0.012)$.

Durante el tiempo del estudio se encontró que el $54.5 \%$ de los reportes de pacientes con infecciones vaginales correspondían a mujeres embarazadas. El mayor número de reportes se debió a vaginitis candidiásica $(9.7 \%)$, en contraste con el $5.4 \%$ reportado para las mujeres no embarazadas. Se encontró además, que en las mujeres embarazadas, el riesgo de adquirir infecciones vaginales se incrementó en 67 veces en forma significativa $(20.8 \% ; \mathrm{OR}=67.016 ; \mathrm{p}=0.000)$. Aunque entre las mujeres no embarazadas la presencia de infecciones vaginales es significativa $(P=0.000)$, en este grupo no existe riesgo de desarrollar esta patología (tabla $3)$.

El 69.4\% de mujeres embarazadas con infecciones vaginales se encontró entre los 20 y 40 años, seguido de las adolescentes (23\%). Entre las comorbilidades encontradas en este grupo se determinó la ITU $(2.4 \%)$, todas ellas en mujeres con vaginitis candidiásica.

\section{DISCUSIÓN}

Las infecciones del aparato genital femenino constituyen un problema de salud pública, tanto en las mujeres de países industrializados como de países en vías de desarrollo, no sólo por el gasto económico al tratarlas, sino porque sus 
Tabla 3. Distribución de vaginitis diagnosticadas en mujeres embarazadas y su relación con el riesgo del embarazo, $n=534$

\begin{tabular}{lcccccccc}
\hline Diagnóstico & \multicolumn{3}{c}{ No embarazadas } & \multicolumn{5}{c}{ Embarazadas } \\
\hline & $\mathrm{n}$ & OR & $\begin{array}{c}\text { IC } \\
\text { Min Máx }\end{array}$ & $\mathrm{p}$ & $\mathrm{n}$ & OR & IC & $\mathrm{p}$ \\
& $(\%)$ & & Mo) & & Min Máx & \\
\hline Vaginosis & 54 & 1.438 & $0.934-2.213$ & 0.098 & 48 & 0.675 & $0.439-1.039$ & 0.073 \\
Bacteriana & $(10.1)$ & & & & $(9)$ & & & \\
\hline Vaginitis & 31 & 0.684 & $0.423-1.108$ & 0.121 & 52 & 1.488 & $0.919-2.409$ & 0.104 \\
Candidiásica & $(5.8)$ & & & & $(9.7)$ & & & \\
\hline Cervicitis & 12 & 1.343 & $0.582-3.101$ & 0.488 & 11 & 0.756 & $0.328-1.746$ & 0.512 \\
& $(2.2)$ & & & & $(2.1)$ & & & \\
\hline Total & 97 & 0.278 & $0.212-0.366$ & 0.000 & 111 & 67.016 & $16.32-275.08$ & 0.000 \\
& $(18.2)$ & & & & $(20.8)$ & & & \\
\hline
\end{tabular}

$\mathrm{OR}=$ Odds Ratio

$\mathrm{P}=$ Error estimado con índice de confianza del 95\%

complicaciones figuran entre las causas más importantes de enfermedad y muerte, especialmente, en mujeres que habitan las regiones pobres del mundo $(6,8,12,15)$. Estas infecciones afectan a mujeres en todos los grupos de edad, principalmente en edad reproductiva $(4,6,12)$. La edad promedio de mujeres con infecciones vaginales obtenida en este estudio fue de 24 años, en coherencia con el resultado obtenido en un estudio realizado en Bogotá, Colombia, en el que la edad promedio fue de 27 años (20). En el presente estudio, el 81,8\% de las pacientes que consultaron por infecciones vaginales se encontraban en un rango de edad entre 20 y 40 años, lo que concuerda con otros estudios realizados en regiones de África (21) y en otras ciudades de Colombia $(12,22)$, incluso en regiones de Brasil se reportan prevalencias mayores del 50\% entre las adolescentes (15). Por lo que el seguimiento y educación en cuanto a las enfermedades de transmisión sexual debe iniciarse desde temprana edad, especialmente por los riesgos que desencadenan en el desarrollo de infertilidad y de otras enfermedades $(16,17)$.

Se ha establecido que la menstruación y las relaciones sexuales son los factores que más influyen en la microbiota vaginal (2). El sangrado menstrual $\mathrm{y}$ el semen tienen un $\mathrm{pH}$ neutro o ligeramente alcalino, que disminuye la población de lactobacilos. Además, el arrastre de la flora normal por el flujo menstrual, el uso de tampones higiénicos y las relaciones sexuales favorecen la proliferación de $G$. vaginalis, $C$. albicans y de los microorganismos entéricos $\mathrm{y}$, como consecuencia, la aparición esporádica de vaginosis y vaginitis $(1,2,23)$.

Las tres causas más comunes de infecciones vaginales reportadas en estudios previos realizados en Colombia, son, vaginosis bacteriana (40-50 \%), seguida por candidiasis $(20-25 \%)$, y tricomoniasis $(15-20 \%)(12,20,22)$. En este estudio se determinó un $18.5 \%$ de vaginosis bacteriana, $15.7 \%$ de vaginitis candidiásica y sólo el $2.6 \%$ se debió a infección por T. vaginalis. Esta tendencia ha sido también reportada en otras regiones de Latinoamérica como Brasil (15) y Chile (24). La vaginosis bacteriana es también la más frecuente llegando a reportarse una prevalencia del $36 \%$ en mujeres latinas y afroamericanas en los Estados Unidos (23) y en mujeres africanas (6). En otro estudio realizado en Bogotá, la vaginosis bacteriana representó el $39.6 \%$ de los casos de infecciones vaginales seguido por candidiasis con un 11\% (25). 
Sin embargo, en este estudio se determinó que el $58 \%$ de las consultas fueron debidas a infecciones mixtas con predominio de bacilos Gram negativos, cocos Gram positivos, Corynebacterias $y$ Lactobacillus spp; en concordancia con los resultados obtenidos en el estudio de Kadir y col, la presencia de aislamientos de E. coli presentó una frecuencia del $13.6 \%$, Klebsiella ssp del 2.0\%, además de otras enterobacterias menos frecuentes como Citrobacter spp, Enterobacter spp, Pseudomonas spp, M. morganii y P. mirabilis (26). La OMS señala que más del $50 \%$ de casos de infecciones vaginales son causadas por infecciones mixtas y en varios casos, las mujeres a pesar del estudio bacteriológico quedan sin diagnóstico, como es el caso de nuestro reporte (17).

En este sentido también se ha establecido que aunque la vaginosis bacteriana, la candidiasis y la tricomoniasis son las más reportadas en este tipo de infecciones resultan ser insuficientes para explicar todos los síntomas clínicos, las fallas en el tratamiento y las recaídas que se observan en las pacientes. Es importante considerar a los microorganismos del ecosistema vaginal, estos pueden causar molestias cuando su equilibrio se altera, como lo refieren Sánchez y col, los microorganismos que inicialmente fueron saprófitos ya no lo son y presentan un comportamiento patógeno en el momento de la consulta, y así junto a Candida spp. y G. vaginalis, la presencia de E. coli y $S$. epidermidis deben ser tratadas (27). Por lo que es recomendable que en los estudios microbiológicos no se reporten dichos microorganismos como normales o simplemente se obvien (1).

Es importante destacar que las mujeres entre las edades de 41 a 64 años presentaron mayor riesgo de adquirir vaginosis bacteriana $(2.1 \%$, OR:2.575, $\mathrm{p}=0.001$ ). Este hallazgo probablemente se deba a que la disminución en la producción de estrógenos se acompaña de una gran reducción del volumen de exudado que baña la superficie vaginal y consecuentemente de los nutrientes, provocando una reducción de la densidad de microorganismos residentes (Lactobacillus sp.), favoreciendo la colonización de la vagina por patógenos $(28,29)$.

En relación a complicaciones asociadas, los resultados de este estudio coinciden con la literatura revisada, ya que se presentó una asociación entre el diagnóstico de vaginosis bacteriana y un mayor riesgo de desarrollar neoplasias; Mendoza y col encontraron una relación entre la vaginosis bacteriana y las lesiones cervicales precancerosas, con un OR estimado de 1.51 (IC del $95 \%$ IC, 1.241.83) (30), y Gillet y col en un metaanálisis encontraron asociación con la neoplasia intraepitelial cervical en el 5\% de las mujeres con vaginosis bacteriana (31), al igual que en otros reportes realizados en Colombia (32). Otra complicación ginecológica con la que se encontró asociación la vaginosis bacteriana fue con la EPI. Esta enfermedad es una de las consecuencias más graves de las infecciones vaginales en la mujer y ocurre cuando una infección del tracto genital inferior (cuello uterino o vagina) o microorganismos externos alcanzan el tracto genital superior (útero, trompas de Falopio, ovarios y estructuras adyacentes) (16). La infección puede generalizarse y poner en riesgo la vida, y el daño tisular y la cicatrización resultante pueden ocasionar infertilidad, dolor pélvico crónico y mayor riesgo de embarazo ectópico.

Las mujeres en estado de embarazo no están exentas de sufrir estas infecciones, por el contrario, la depresión inmunológica que lo caracteriza, constituye un elemento facilitador o predisponerte a un mayor número de estas infecciones $(3,4)$. En el embarazo estas infecciones afectan la unidad fetoplacentaria, llevando a complicaciones como, parto pretérmino, ruptura prematura de membranas y recién nacido de bajo peso; e incluso complicaciones maternas, como corioamnionitis e infección puerperal (4). Las mujeres embarazadas diagnosticadas con infecciones vaginales representaron el $20,8 \%$, con un promedio de edad de 22 años, el mayor número de mujeres embarazadas se concentró entre los 20 y 40 años, las menores de 20 años representaron el $23 \%$, en contraste al estudio realizado por Zemenu y col, en Etiopia donde el 85.7 
$\%$ de gestantes tuvieron infección vaginal y el 57.1

$\%$ eran menores de 20 años (33). En Colombia, los reportes en otras ciudades señalan un $82 \%$ de infecciones en este grupo con un rango de edad entre 13 y 45 años (34).

La vaginitis candidiásica y la vaginosis bacteriana fueron las más prevalentes con un $9.6 \%$ y el $10.1 \%$, respectivamente. En la literatura, los datos epidemiológicos muestran prevalencias cercanas al $29 \%$ de vaginosis bacteriana, seguida por vaginitis por $C$. albicans del $21 \%$ y por $T$. vaginalis del 4 $\%$, en el caso de las mujeres inglesas (4). En Chile, se reporta una prevalencia más alta de vaginosis bacteriana en mujeres embarazadas que corresponde al $20.8 \%$ (3).

La vaginosis bacteriana se asocia con la presencia de endometritis posterior a cesáreas $u$ otros procedimientos obstétricos, por lo que se debe dar tratamiento a las mujeres con factores de riesgo, aunque estén asintomáticas. Las complicaciones obstétricas asociadas a la vaginosis bacteriana son: parto pretérmino, corioamnionitis, endometritis puerperal y ruptura prematura de membranas principalmente $(4,34)$. Existen estudios que asocian la vaginosis bacteriana durante el embarazo con un resultado perinatal adverso, en particular un mayor riesgo de parto prematuro. El fuerte vínculo entre la vaginosis bacteriana y el parto prematuro puede ser la causa de este en las mujeres que la padecen (34).

En el caso de la infección por $C$. albicans se consideran factores de riesgo inherentes al huésped, diabetes mellitus no controlada, inmunodeficiencia, hiperestrogenismo y alteración de la flora vaginal normal secundaria al uso de antibióticos de amplio espectro (10). El desarrollo de vulvovaginitis por $T$. vaginalis en la mujer embarazada presenta como factores de riesgo, historia sexual de cambio de pareja con más de una pareja sexual en el último año, o diagnóstico de infección por clamidias en los últimos doce meses $(13,35)$.

Con respecto a las características fenotípicas de los exudados vaginales se evidenciaron frecuentemente frotis vaginales de pacientes con exudados blanquecinos con la presencia de blastoconidias, compatible con candidiasis, y en raras ocasiones se evidenció la presencia de células clave; mientras que en los exudados amarillos se detectaron frecuentemente tricomonas. Estos resultados coinciden con lo registrado en la literatura, la manifestación principal de la vaginitis por $T$. vaginalis es la leucorrea amarillenta, que casi invariablemente se asocia al dolor vaginal y puede mostrar disuria (14). La leucorrea blanco grisácea con presencia de células clave fue la manifestación principal de la vaginosis bacteriana (18). Sin embargo, en este estudio se encontró que el 1,6\% de los casos reportados con infección por T. vaginalis, presentaron exudado blanquecino, lo que no es compactible con los reportes en la literatura, la leucorrea blanquecina, el prurito y la disuria que se asocia frecuentemente con la presencia de microrganismos del género Candida $s p .(5,12)$.

Un aspecto encontrado en este estudio fue que 324 de los registros resultaron negativos, lo que pudo deberse a la presencia de otros agentes biológicos no diagnosticados causantes de infección ginecológica, entre los que hay que considerar a Neisseria gonorroheae, Chlamydia trachomatis, Mycoplasma spp. y Herpes Virus tipo 2, entre otros, que explican estas entidades clínicas en dichas mujeres $(25,35)$. Los reportes epidemiológicos evidencian un incremento de infecciones debidas a C. trachomatis y Ureaplasma. urealyticum en las últimas décadas, llegando a prevalencias que alcanzan el $71 \%$ para $M$. hominis, $23.2 \%$ para $U$. parvum y $5.8 \%$ en el caso de $U$. urealyticum, y son responsables de casi el $60 \%$ de las uretritis no gonocóccicas en Brasil y Chile $(37,38)$; siendo el grupo de pacientes entre los 18-28 años las que presentan la más alta prevalencia $13.7 \%$ Estos gérmenes se asocian con obstrucción tubárica, hidrosálpinx y síndrome adherencial (37). La OMS, señala la necesidad de aplicar técnicas de amplificación de ácidos nucleicos para identificar a C. trachomatis o cultivo para el aislamiento de Mycoplasma spp con el fin de descartar infecciones por estos microrganismos en extendidos reportados como negativos o presencia de flora normal en mujeres sintomáticas como los obtenidos en el 58\% de los resultados de este estudio. 
En conclusión, este estudio demuestra que la vaginosis bacteriana y la vaginitis candidiásica son las infecciones vaginales que más afectan a las mujeres en edad reproductiva y sus principales complicaciones derivan en enfermedad pélvica inflamatoria, hemorragias y neoplasias, especialmente, en mujeres de estratos socioeconómicos bajos. Aunque el un poco más de la mitad de los frotis vaginales presentaron exudado negativo para las etiologías de mayor prevalencia expuestas, no se descarta la etiología por otros patógenos (C. trachomatis y Mycoplasma Spp) que requieren métodos diagnósticos más sofisticados para detectarlos.

CONFLICTOS DE INTERESES: Los autores no declaran conflictos de interés.

\section{REFERENCIAS}

1. Gajer P, Brotman RM, Bai G, Sakamoto J, Schutte UM, Zhong X, Koenig SS, Fu L, Ma ZS, Zhou X, Abdo Z, Forney LJ, Ravel J. Temporal dynamics of the human vaginal microbiota.Sci Transl Med. 2012;4(132):132ra52

2. Lambert JA, Kalra A, Dodge CT, John S, Sobel JD, Akin RA. Novel PCR-Based Methods Enhance Characterization of Vaginal Microbiota in a Bacterial Vaginosis Patient before and after Treatment. Novel PCR-Based Methods Enhance Characterization of Vaginal Microbiota in a Bacterial Vaginosis Patient before and after Treatment, Appl Environ Microbiol. 2013;79(13): 4181-85.

3. Pradenas M. InfeccIones cérvico vagInales y embarazo, Rev. Med. Clin. Condes. 2014;25(6) 92535 .

4. Gupta NK, Bowman CA. Managing sexually transmitted infections in pregnant women, Womens Health (Lond Engl). 2012;8(3):313-21.

5. Saona U. Vaginitis. Ampliando el espectro diagnóstico, Rev Per Ginecol Obstet 2012;53:153-58.

6. Narayankhedkar A, Hodiwala A, Mane A. Clinicoetiological Characterization of Infectious Vaginitis amongst Women of Reproductive Age Group from Navi Mumbai, India. J Sex Transm Dis. 2015; 2015: 817092 .
7. Trejos R. Vaginosis Bacteriana, Rev Med Costa Rica y Centroamérica. 2012; LXIX (602):183-87.

8. Srinivasan S, Hoffman NG, Morgan MT, Matsen FA, Fiedler TL, Hall RW, Ross FJ, McCoy CO, Bumgarner R, Marrazzo JM, Fredricks DN. Bacterial communities in women with bacterial vaginosis: high resolution phylogenetic analyses reveal relationships of microbiota to clinical criteria. PLoS One. 2012;7(6): e37818

9. Cassone A. Vulvovaginal Candida albicans infections: pathogenesis, immunity and vaccine prospects. BJOG. 2015; 122:785-94.

10. Kalkanci A, Güzel AB, Khalil II, Aydin M, Ilkit M, Kuştimur S. Yeast vaginitis during pregnancy: susceptibility testing of 13 antifungal drugs and boric acid and the detection of four virulence factors, Med Mycol. 2012; 50(6): 585-93.

11. Davies S, Johnson E, White D. How to treat persistent vaginal yeast infection due to species other tan Candida albicans, Sex Transm Infect. 2013; 89: 1656.

12. Morales GI, Yaneth MC. Candidiasis en mujeres en edad reproductiva que asistieron al hospital Eduardo Arredondo Daza en la ciudad de Valledupar, Rev Col Microbiol Trop. 2012; 2: 13-21.

13. Centers for Disease Control. Trichomoniasis. Atlanta (GA): CDC; 2013. Disponible en: http://www.cdc.gov/std/trichomonas/default.htm

14. Coleman JS, Gaydos CA, Witter F. Trichomonas vaginalis vaginitis in obstetrics and gynecology practice: new concepts and controversies, Obstet Gynecol Surv. 2013;68(1):43-50.

15. Mascarenhas RE, Machado MS, Costa e Silva BF, Pimentel RF, Ferreira TT, Leoni FM, et al. Prevalence and risk factors for bacterial vaginosis and other vulvovaginitis in a population of sexually active adolescents from Salvador, Bahia, Brazil, Infect Dis Obstet Gynecol. 2012; 2012: 378640.

16. Wiesenfeld HC, Hillier SL, Meyn LA, Amortegui AJ, Sweet RL. Subclinical pelvic inflammatory disease and infertility, Obstet Gynecol 2012;120:37-43. 
17. Organización Mundial de la Salud. Infecciones de transmisión sexual; 2013. Disponible en: http://www.who.int/mediacentre/ factsheets/fs110/es/

18. Amsel R, Totten PA, Spiegel CA, Chen KC, Eschenbach D, Holmes KK. Nonspecific vaginitis. Diagnostic criteria and microbial and epidemiologic associations. Am J Med. 1983;74 (1):14-22.

19. Lang TA, Altman DG. Basic statistical reporting for articles published in biomedical journals: the "Statistical Analyses and Methods in the Published Literature" or the SAMPL Guidelines. Int J Nurs Stud. 2015;52(1):5-9.

20. Ángel E, Rodríguez A, Núñez LM, Moyano LF, González P, Osorio E, et al. The prevalence of and factors associated with $C$. trachomatis, $N$. gonorrheae, T. vaginalis, C. albicans infection, syphilis, HIV and bacterial vaginosis in females suffering lower genital tract infection symptoms in three healthcare attention sites in Bogotá, Colombia, 2010. Rev Colomb Obstet Ginecol. 2012; 63(1):14-24.

21. NzomoJ, Waiyaki P, Waihenya R. Bacterial Vaginosis and Correlates in Women of Reproductive Age in Thika, Kenya, Adv Microbiol. 2013;3:249-54.

22. Cardona JA, Herrera D, Valencia M. Prevalencia de resultado positivo de la citología para vaginosis bacteriana, candidiasis y tricomoniasis en una Empresa Social del Estado de Medellín (Colombia), 2010-2012. Rev Colomb Obstet Ginecol. 2014; 65(3):206-14.

23. Brown JM, Hess KL, Brown S, Murphy C, Waldman AL, Hezareh M. Intravaginal practices and risk of bacterial vaginosis and candidiasis infection among a cohort of women in the United States. Obstet Gynecol. 2013;121:773-80.

24. Heredia R, Agudelo C, Castañeda E. Prevalencia de los agentes etiológicos de la vaginitis y la cervicitis en pacientes de consulta ginecológica general. Rev Chil Obstet Ginecol. 2012;32:17-36.

25. Müller EA, Rodríguez A, Núñez L, Moyano LF, González P, Osorio E y col. Prevalencia y factores asociados a la infección por $C$. trachomatis, $N$. gonorrheae, $T$. vaginalis, $C$. albicans, sífilis, VIH y vaginosis bacteriana en mujeres con síntomas de infección vaginal en tres sitios de atención de Bogotá, Colombia, 2010, Rev. Colomb. Obstet. Ginecol. 2012; 63(1):14-24.
26. Kadir MA, Sulyman MA, Dawood IS, Shams Eldin S, Trichomonas vaginalis and associated microorganisms in women with vaginal discharge in Kerkuk-Iraq. Ankara Med J. 2014.14(3):91-99.

27. Sánchez J, Rebollo M, Paulin J. Cambios en el pH vagina en pacientes con infec- ción cérvico-vaginal. Enferm Infecc Microbiol Clin. 2013;33:61-5.

28. Mitchell C, Marrazzo J. Bacterial vaginosis and the cervicovaginal immune response. Am J Reprod Immunol. 2014;71(6): 555-63.

29. Murphy K, Mitchell CM. The Interplay of Host Immunity, Environment and the Risk of Bacterial Vaginosis and Associated Reproductive Health Outcomes. J Infect Dis. 2016; 214: S29 -S35.

30. Mendoza L, Alfonso L, Pedroza M, Micolta P, Ramirez A, Acuña M, et al. Prevalencia de lesiones de bajo y alto grado de cuello uterino en una ciudad colombiana. Rev Chil Obstet Ginecol. 2012;77(2):129-36.

31. Gillet E, Meys JF, Verstraelen H, Verhelst R, De Sutter $\mathrm{P}$, Temmerman M. Association between bacterial vaginosis and cervical intraepithelial neoplasia: systematic review and meta-analysis. PLoS One. 2012;7(10): e45201.

32. Zapata JF, López L, Tirado AF, Gómez LJ, González JD, Rodríguez MC, et al. Frecuencia de infecciones vaginales y lesiones intraepiteliales en las usuarias de los laboratorios clínicos de Dinámica IPS de la ciudad de Medellín (Colombia), 2005-2010: estudio descriptive. Arch Med (Manizales). 2015;15 (2):24149.

33. Zemenu M, Yimtubezinash $\mathrm{W}$, As rat D, Yigeremu M. Comparison of Clinical and Gram Stain Diagnosis Methods of Bacterial Vaginosis Among Pregnant Women in Ethiopia. J Clin Diagn Res. 2013;7(12):2701-03.

34. Salas N, Ramírez JF, Ruiz B, Torres E, Jaramillo LN, Gómez-Marín JE. Prevalencia de microorganismos asociados a infecciones vaginales en 230 mujeres gestantes y no gestantes sintomáticas del Centro de Salud La Milagrosa en el municipio de Armenia (Colombia). Rev Colomb Obstet Ginecol. 2009; 60(2): $135-42$. 
35. Blatt A, Lieberman J, Hoover D, Kaufman H. Chlamydial and gonococcal testing during pregnancy in the United States. Am J Obstet Gynecol. 2012;207(1):55.e1-8

36. Díaz L, Cabrera LE, Fernández T, Ibáñez I, Torres Y, Obregón Y, et al. Frequency and antimicrobial sensitivity of Ureaplasma urealyticum and Mycoplasma hominis in patients with vaginal discharge. MEDICC Rev.2013; 15(4):45-7

37. Urdaneta J, Cantillo E, Alarcón A, Karame A, Salazar J, Romero R. Infertilidad tubárica e infección genital por Chlamydia trachomatis-Ureaplasma urealyticum, Rev Chil Obstet Ginecol. 2013;78(1):32 -43.

38. Costoya A, Morales F, Borda P, Vargas R, Fuhrer J, Salgado N, et al. Mycoplasmateceae species are not found in Fallopian tubes of women with tuboperitoneal infertility,Braz. J Infect Dis. 2012;16:273-8. 1. 